\title{
EMPREGO DE COMPOSTOS ORGANOMETÁlICOS MONONUCLEARES DE PALÁDIO(II) NA ATIVAÇÃo DE MACRÓFAGOS PERITONEAIS DE CAMUNDONGOS
}

Eduardo Tonon de Almeida*, Antonio Eduardo Mauro*, Anderson Martinez Santana e Adelino Vieira de Godoy Netto Departamento de Química Geral e Inorgânica, Instituto de Química de Araraquara, Universidade Estadual Paulista, CP 355, 14800-900 Araraquara - SP

Iracilda Zeppone Carlos

Departamento de Análises Clínicas, Faculdade de Ciências Farmacêuticas de Araraquara, Universidade Estadual Paulista, CP 502, 14801-902 Araraquara - SP

Recebido em 3/3/04; aceito em 26/10/04; publicado na web em 17/2/05

ACTIVATION OF MICE PERITONEAL MACROPHAGES BY PALLADIUM(II) ORGANOMETALLIC MONONUCLEAR COMPOUNDS. The immune responses are mediated by a variety of cells that, when activated, produce a number of molecules. Macrophages are the first cells to take part in the immune response releasing many compounds in the extracellular environment such as $\mathrm{H}_{2} \mathrm{O}_{2}$. Taking into account this aspect we evaluated the activation of an immunological system, in vitro, by determining the $\mathrm{H}_{2} \mathrm{O}_{2}$ released in cultures of peritoneal macrophage cells from Swiss mice in the presence of organopalladated compounds of the type $[\mathrm{Pd}(\mathrm{dmba})(\mathrm{X})(\mathrm{dppp})], \mathrm{dmba}=N, N$-dimethylbenzylamine, dppp = 1,3-bis(diphenylphosphine)propane, $\mathrm{X}=\mathrm{Cl}, \mathrm{N}_{3}, \mathrm{NCO}, \mathrm{NCS}$. An excellent activation of macrophages by the $[\mathrm{Pd}(\mathrm{dmba})(\mathrm{X})(\mathrm{dppp})]$ compounds was observed and the influence of the $\mathrm{X}$ ligand on the immune response could be verified.

Keywords: palladium(II) complexes; macrophages; hydrogen peroxide.

\section{INTRODUÇÃO}

Os complexos organometálicos de paládio(II) são extensivamente investigados há décadas e têm encontrado diversas aplicações como em catálise homogênea ${ }^{1}$, química supramolecular ${ }^{2}$, na área tecnológica como materiais líquido-cristalinos ${ }^{3}$, na área médica como agentes anti-tumorais ${ }^{4}$, entre outras. Estes compostos também apresentam uma grande reatividade frente a ligantes orgânicos e inorgânicos originando, assim, espécies com uma rica variedade estrutural. Na química medicinal, destacam-se nos últimos anos os derivados do $\mathrm{Pd}(\mathrm{II})$, sobre os quais há muitas citações contemplando espécies organopaladadas ${ }^{5}$ mono, bi e polinucleares com propriedades biológicas ${ }^{6}$. Por ex., o organopaladado contendo o ligante derivado da tiosemicarbazona ${ }^{6},\left[\mathrm{Pd}\left(\mathrm{C}_{9} \mathrm{H}_{11} \mathrm{~N}_{3} \mathrm{~S}\right) \mathrm{Cl}_{2}\right]$, apresentou atividade citotóxica in vitro frente a certos tipos de células cancerosas, sendo a mesma superior àquela do análogo com platina(II).

Recentemente, organometálicos de paládio(II) contendo as difosfinas 1,3-bis(difenilfosfina)propano $(d p p p)^{7}$ e 1,4 bis(difenilfosfina)butano $(d p p b)$, Figura 1, mostraram uma atividade biológica promissora frente à células cancerosas cultivadas in vitro ${ }^{8}$.

Embora seja constatada, atualmente, uma forte tendência na investigação da atividade biológica envolvendo interações entre complexos inorgânicos com DNA e RNA, verifica-se, por outro lado, serem escassos os estudos tratando da estimulação de macrófagos do sistema imunológico por estas espécies químicas.

Os macrófagos constituem um dos principais componentes do sistema imunológico e são as primeiras células a serem ativadas para participar de uma resposta imunológica, propriamente dita,

*e-mail: mauro@iq.unesp.br

\# Endereço atual: Curso de Química, Universidade Católica de Brasília, CP 5490, 71966-700 Brasília - DF

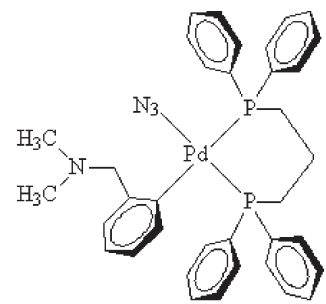

(a)

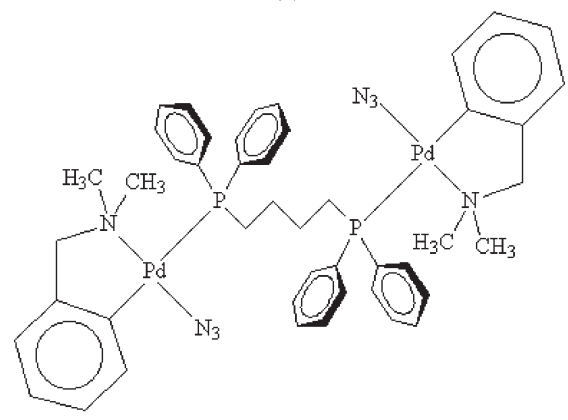

(b)

Figura 1. Fórmulas estruturais planas dos organometálicos $\left[P d(d m b a)\left(N_{3}\right)(d p p p)\right]$ (a) e $\left[P d_{2}(d m b a)_{2}\left(N_{3}\right)(\mu-d p p b)\right](b), d m b a=N, N-$ dimetilbenzilamina, dppp $=1,3$-bis(difenilfosfina)propano e $d p p b=1,4$ bis(difenilfosfina)butano

quando o organismo é exposto a fatores exógenos, como por exemplo bactérias, vírus, fungos, venenos, dentre outros. Os macrófagos podem ser ativados, portanto, por uma variedade de estímulos e suas principais funções incluem fagocitose das partículas estranhas, produção de citocinas e de mediadores químicos ${ }^{9}$, como o $\mathrm{H}_{2} \mathrm{O}_{2}$ e o NO. Após exposição a um estímulo adequado, como à bactérias, os fagócitos polimorfonucleares e mononucleares sofrem 
uma mudança em seu metabolismo. Nesse caso ocorre um aumento no consumo de oxigênio, que chega a atingir níveis 50 vezes maiores que os observados quando as células estão em repouso. Concomitantemente nota-se substancial aumento na oxidação da glicose, via hexose monofosfato, com produção de NADPH. A NADPH (adenina-difosfato-nicotinamida) oxidase atua como doadora de um elétron, promovendo a redução do $\mathrm{O}_{2} \mathrm{a} \mathrm{O}_{2}^{-}$e este, por dismutação espontânea ou através da enzima superóxido dismutase (SOD), é transformado em $\mathrm{H}_{2} \mathrm{O}_{2}{ }^{10}$, conforme mostram as Equações 1 e 2 .

$2 \mathrm{O}_{2}+\mathrm{NADPH} \longrightarrow 2 \mathrm{O}_{2}^{-}+\mathrm{NADP}^{+}+\mathrm{H}^{+}$

$2 \mathrm{O}_{2}^{-}+2 \mathrm{H}^{+} \stackrel{\mathrm{SOD}}{\longrightarrow} \mathrm{H}_{2} \mathrm{O}_{2}+\mathrm{O}_{2}$

A geração de $\mathrm{H}_{2} \mathrm{O}_{2}$ é um processo celular natural, resultante de várias reações específicas essenciais à atividade celular. No entanto, a liberação de quantidades consideráveis de $\mathrm{H}_{2} \mathrm{O}_{2}$ induz a quebra de fita de DNA e/ou perturbação no citoesqueleto da membrana, levando à morte celular ${ }^{11,12}$.

Compostos capazes de provocar a liberação de $\mathrm{H}_{2} \mathrm{O}_{2}$ por macrófagos, em pequenas concentrações, são importantes, pois auxiliam na resposta imunológica como organo-comunicadores. Um método eficiente para se quantificar o peróxido de hidrogênio liberado por células cultivadas in vitro é baseado na ação da enzima denominada peroxidase de raiz forte ("horseradish peroxidase - HRPO"), que catalisa a oxidação do vermelho de fenol pelo $\mathrm{H}_{2} \mathrm{O}_{2}$. Esta reação origina um composto amarelo que, com a elevação do $\mathrm{pH}$ do meio a 12,5, adquire uma coloração violeta, absorvendo em 620 $\mathrm{nm}$ e permitindo, então, determinar a concentração de $\mathrm{H}_{2} \mathrm{O}_{2}$ produzida por macrófagos ativados em cultura de células.

Considerando o exposto, o presente trabalho teve como objetivo investigar a influência da série de complexos do tipo [Pd(dmba)X(dppp)], X= Cl (1), $\mathrm{N}_{3}(\mathbf{2}), \operatorname{NCO}(\mathbf{3})$ e NCS (4), na liberação de peróxido de hidrogênio por macrófagos em cultura de células peritoneais de camundongos in vitro, empregando-se o método espectrofotométrico ${ }^{11}$ na sua determinação. Foi constatada a influência dos substituintes $\mathrm{X}=\mathrm{Cl}, \mathrm{N}_{3}$, NCO e NCS na concentração de $\mathrm{H}_{2} \mathrm{O}_{2}$ liberada, e os dados são promissores quanto ao futuro emprego destes compostos de $\mathrm{Pd}(\mathrm{II})$ como organo-comunicadores, auxiliando numa resposta biológica.

\section{PARTE EXPERIMENTAL}

\section{Preparação dos compostos}

Os compostos $[\mathrm{Pd}(\mathrm{dmba})(\mathrm{Cl})(\mathrm{dppp})](\mathbf{1})^{13},\left[\mathrm{Pd}(\mathrm{dmba})\left(\mathrm{N}_{3}\right)\right.$ (dppp)] (2) ${ }^{8}$ foram preparados como descrito na literatura.

Sinteses dos compostos $[\mathrm{Pd}(\mathrm{dmba})(\mathrm{X})(\mathrm{dppp})]\{\mathrm{X}=\mathrm{NCO}(\mathbf{3}), \mathrm{NCS}(\mathbf{4})\}$

Em um erlenmeyer contendo $0,35 \mathrm{mmol}$ dos precursores $[\mathrm{Pd}(\mathrm{dmba})(\mu-\mathrm{X})]_{2}\{0,20 \mathrm{~g}(\mathrm{X}=\mathrm{NCO}) ; 0,21 \mathrm{~g}(\mathrm{X}=\mathrm{NCS})\}$, suspensos em $30 \mathrm{~mL}$ de acetona e sob agitação magnética, foram adicionados 0,60 g (0,70 mmol) de 1,3-bis(difenilfosfina)propano. Manteve-se a mistura reacional sob agitação constante, à temperatura ambiente, por $1 \mathrm{~h}$. Evaporou-se lentamente o solvente da solução formada, sem aquecimento, até quase a completa secura. Adicionou-se então, $10 \mathrm{~mL}$ de diclorometano, filtraram-se as impurezas e à solução final adicionou-se pentano, resultando na precipitação de um sólido claro. Este sólido foi lavado com pentano e seco sob vácuo.

Composto 3, Análise elementar: \% calc. para $\mathrm{C}_{37} \mathrm{H}_{38} \mathrm{~N}_{2} \mathrm{OP}_{2} \mathrm{Pd}$ (\% enc.): C: 63,94(63,81); H: 5,51(5,40); N: 4,03(3,98). Temperatura de fusão: $145,7{ }^{\circ} \mathrm{C}$. Rendimento: $85 \%$.
Composto 4, Análise elementar: \% calc. para $\mathrm{C}_{37} \mathrm{H}_{38} \mathrm{~N}_{2} \mathrm{SP}_{2} \mathrm{Pd}$ (\% enc.): C: 62,49(62,28); H: 5,39(5,27); N: 3,94(4,09). Temperatura de fusão: $140,3{ }^{\circ} \mathrm{C}$. Rendimento: $90 \%$.

\section{Técnicas experimentais}

Todos os complexos sintetizados e seus respectivos precursores $^{14}$ foram caracterizados por técnicas de IV, RMN de ${ }^{1} \mathrm{He}{ }^{13} \mathrm{C}\left\{{ }^{1} \mathrm{H}\right\}$, análise elementar de $\mathrm{C}, \mathrm{H}, \mathrm{N}$ e $\mathrm{S}$ e ponto de fusão.

No que concerne aos novos compostos [Pd(dmba)(NCO)(dppp)] (3) e $[\mathrm{Pd}(\mathrm{dmba})(\mathrm{NCS})(\mathrm{dppp})]$ (4), as análises quantitativas dos elementos carbono, hidrogênio e nitrogênio foram efetuadas no analisador automático EA 1110 CHNS-O, da CE-Instruments.

Os espectros de absorção na região do IV foram obtidos em um espectrofotômetro Nicolet FT-IR Impact 400, no intervalo de 4000 a $400 \mathrm{~cm}^{-1}$, com resolução de $4 \mathrm{~cm}^{-1}$, em pastilhas de $\mathrm{KBr}$.

Os espectros de RMN de ${ }^{1} \mathrm{H}$ e ${ }^{13} \mathrm{C}\left\{{ }^{1} \mathrm{H}\right\}$ foram obtidos em um espectrofotômetro Varian modelo Inova $500 \mathrm{MHz}$, empregando-se as frequências de $500 \mathrm{MHz}$ para hidrogênio e $125 \mathrm{MHz}$ para carbono, respectivamente. Foram empregados como solvente clorofórmio deuterado $\left(\mathrm{CDCl}_{3}-d\right)$ e como padrão interno o tetrametilsilano (TMS).

As temperaturas de fusão foram medidas utilizando-se um aparelho Mettler modelo PF-2.

\section{Ensaios biológicos}

\section{Liberação de $\mathrm{H}_{2} \mathrm{O}_{2}$ por macrófagos}

Animais - Foram utilizados camundongos machos, tipo Swiss, com peso variando entre 18 a $25 \mathrm{~g}$, todos fornecidos pelo Biotério da FCF - Unesp.

Obtenção de macrófagos e determinação de $\mathrm{H}_{2} \underline{O}_{2}$ - Foi empregado um método de cultura in vitro para se quantificar o composto resultante da oxidação do vermelho de fenol pelo peróxido de hidrogênio, devido à liberação do oxigênio radicalar do $\mathrm{H}_{2} \mathrm{O}_{2}$. Este método, conforme descrito na literatura ${ }^{10,15-17}$, consiste na avaliação do "burst" respiratório, quantificando-se o peróxido de hidrogênio liberado por macrófagos, quando em contato com substâncias consideradas estranhas ao organismo.

Procedimentos - Os animais foram previamente inoculados por via intraperitonial, com 3,0 mL de tioglicolato de sódio a 3\%. Após decorridos 3 - 4 dias de estímulo, eles foram sacrificados por deslocamento cervical e, posteriormente à morte e exposição do peritônio, abriu-se a pele do animal, inoculou-se $5,0 \mathrm{~mL}$ de solução salina tamponada de fosfato $\mathrm{pH}$ 7,2 estéril, realizando-se leve massagem manual no mesmo.

As células foram colhidas do peritônio com a mesma seringa e dispensadas em tubo cônico estéril para o preparo da suspensão celular. Esta suspensão foi centrifugada de 3 a 4 vezes a 2000 rpm durante $5 \mathrm{~min}$, separando assim, as células do exsudato peritoneal. Em seguida, as mesmas foram contadas em câmara hemocitométrica tipo Neubauer, ajustando assim, sua concentração a $2.10^{6}$ células por $\mathrm{mL}$ em 9,6 mL de solução tampão fosfato $\mathrm{pH}$ 7,0 (contendo $\mathrm{NaCl}$ com concentração $140 \mathrm{mM}$, tampão fosfato de potássio $\mathrm{pH}$ 7,0 com concentração $10 \mathrm{mM}$ e dextrose com concentração 5,5 $\mathrm{mM}), 0,2 \mathrm{~mL}$ de vermelho de fenol com concentração igual a 0,56 $\mathrm{mM}$ e $0,1 \mathrm{~mL}$ de peroxidase de raiz forte, tipo II, concentração $0,01 \mathrm{mg} / \mathrm{mL}$. Foram transferidas, então, alíquotas de $100 \mu \mathrm{L}$ para microplacas de 96 cavidades. Em algumas cavidades da placa foram adicionados $50 \mu \mathrm{L}$ de uma solução $5 \mathrm{mg} / \mathrm{mL}$ de zimosan, que consiste na parede celular de Saccharomyces cerevisiae e, em outras cavidades, as soluções dos organopaladados em estudo, na concentração de $5 \mathrm{mg} / \mathrm{mL}$, também em solução tampão de fosfato 
pH 7,0. Em outras cavidades foram feitos controles de células contendo somente a suspensão celular em solução tampão de fosfato pH 7,0 e, noutras, foram feitos controles dos reagentes (contendo somente solução de vermelho de fenol e zimosan).

As placas foram incubadas por $60 \mathrm{~min}$ em estufa a $37^{\circ} \mathrm{C}$, sob atmosfera constante de $\mathrm{CO}_{2}$ igual a $5 \%$. Após a incubação, a reação foi interrompida com a adição de $50 \mu \mathrm{L} /$ cavidade de $\mathrm{NaOH} 5 \mathrm{M}$ e, a seguir, foram feitas leituras de absorbância a $620 \mathrm{~nm}$. Os resultados foram expressos em nanomol (nmol) de $\mathrm{H}_{2} \mathrm{O}_{2} / 2.10^{5}$ células, a partir de uma curva padrão previamente estabelecida, constituída de concentrações molares conhecidas de $\mathrm{H}_{2} \mathrm{O}_{2}$ em tampão vermelho de fenol.

Leituras de absorbância - Os dados de absorbância foram obtidos no Espectrofotômetro Multiscan Ascent da Labsystems.

Análise estatística - Foi realizada através do teste- $t$ de Student para amostras não paramétricas de acordo com o programa estatístico Microcal ${ }^{\mathrm{TM}}$ Origin $^{\mathrm{TM}}$ versão $5,0^{18}$. As diferenças foram consideradas significativas quando $\mathrm{p}<0,05$.

\section{RESULTADOS E DISCUSSÃO}

Conforme já colocado anteriormente, os compostos do tipo $[\mathrm{Pd}(\mathrm{dmba})(\mathrm{X})(\mathrm{dppp})], \mathrm{X}=\mathrm{Cl}(\mathbf{1}), \mathrm{N}_{3}(\mathbf{2}), \mathrm{NCO}(\mathbf{3})$ e NCS (4), resultaram da clivagem dos dímeros $[\mathrm{Pd}(\mathrm{dmba})(\mu-\mathrm{X})]_{2}$, em reações com a 1,3-bis(difenilfosfina)propano (dppp). A escolha dos complexos 1-4, para a realização destes testes, foi motivada pelos ótimos resultados obtidos ${ }^{8}$ quando se avaliou a atividade citotóxica do complexo $\left[\mathrm{Pd}(\mathrm{dmba})\left(\mathrm{N}_{3}\right)(\mathrm{dppp})\right](\mathbf{2})$, em células tumorais, cultivadas in vitro, referentes às linhagens HeLa (cólon do útero), Hep2 (orofaringe) e C6 (glioma cerebral).

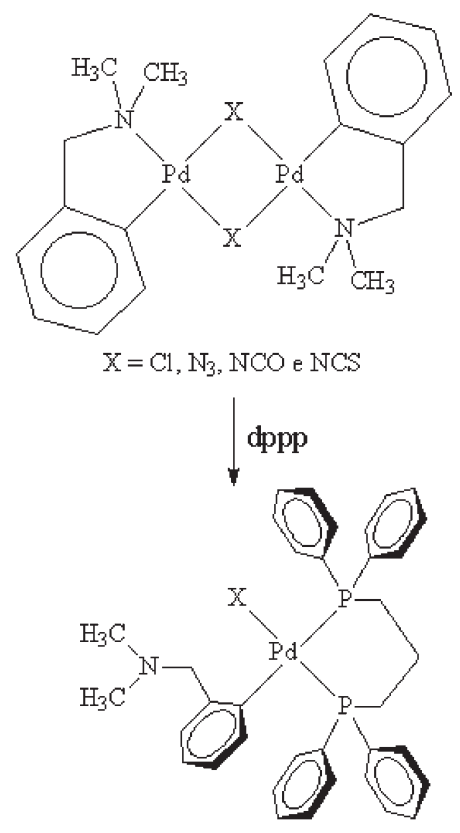

Esquema 1.

O diagnóstico da forma de coordenação terminal dos pseudohaletos foi proposto com base na posição espectral ${ }^{19}$ das bandas atribuídas aos modos de estiramentos assimétrico e simétrico exibidos pelos mesmos nos espectros de absorção na região do IV.

O espectro no IV do composto [Pd(dmba)(NCO)(dppp)] (3) mostra bandas em 3041, 2934 e $2868 \mathrm{~cm}^{-1}$, referentes aos modos $v(\mathrm{CH})_{\text {aromático, }}, v(\mathrm{CH})\left[-\mathrm{N}\left(\mathrm{CH}_{3}\right)_{2}\right]$ e $v(\mathrm{CH})\left[-\mathrm{N}\left(\mathrm{CH}_{2}\right)\right]$, respectivamente, do ligante dmba. A presença das bandas em 2209, 1314, 680 e
$510 \mathrm{~cm}^{-1}$, atribuídas aos modos $v(\mathrm{CN}), v(\mathrm{CO})$ e $\delta(\mathrm{NCO})$, respectivamente, elucida claramente a presença do grupo NCO coordenado de forma terminal ao centro metálico via átomo de nitrogênio. Além destas, as bandas em 1095 e $693 \mathrm{~cm}^{-1}$, associadas aos modos vibracionais $\mathrm{v}(\mathrm{PC})$ e $\delta(\mathrm{C}-\mathrm{P}-\mathrm{C})$ da $d p p p$, confirmam a presença da mesma coordenada ao metal.

De maneira semelhante, o espectro no IV do $[\mathrm{Pd}(\mathrm{dmba})(\mathrm{NCS})$ (dppp)] (4) apresenta bandas em 3039, 2900 e $2859 \mathrm{~cm}^{-1}$, correspondentes aos modos $v(\mathrm{CH})_{\text {aromático, }}, v(\mathrm{CH})\left[-\mathrm{N}\left(\mathrm{CH}_{3}\right)_{2}\right]$ e $v(\mathrm{CH})\left[-\mathrm{N}\left(\mathrm{CH}_{2}\right)\right]$, respectivamente, do ligante dmba. A presença das bandas em 2048, $840,440 \mathrm{~cm}^{-1}$, atribuídas aos modos $v(\mathrm{CN}), v(\mathrm{CS})$ e $\delta(\mathrm{NCS})$, respectivamente, comprovam a coordenação do grupo NCS terminal via átomo de nitrogênio. Cabe mencionar que a coordenação do grupo SCN pelo átomo de nitrogênio no complexo $\mathbf{4}$ está associada à competição entre os ligantes $d p p p$ e o tiocianato pela formação de uma ligação $\pi$ com os orbitais $d$ do paládio. O ligante $d p p p$ realiza ligações $\pi$ mais eficientes que o $S$-tiocianato e, conseqüentemente, tende a monopolizar os orbitais $\pi$-ligantes do paládio. Dessa forma, a estabilidade da ligação $\pi$ envolvendo o átomo de enxofre do grupo tiocianato e o paládio é reduzida, favorecendo então a coordenação do pseudo-haleto via átomo de nitrogênio.

Foram também medidos os espectros de RMN de ${ }^{1} \mathrm{H}$ e ${ }^{13} \mathrm{C}\left\{{ }^{1} \mathrm{H}\right\}$ e os sinais observados foram atribuídos como se segue: RMN de ${ }^{1} \mathrm{H}, \delta$ em ppm: [-N( $\left.\left(\mathrm{CH}_{3}\right)_{2}\right],(\mathbf{1}), 2,27[\mathrm{~s}, 6 \mathrm{H}],(\mathbf{2}), 2,64[\mathrm{~s}, 6 \mathrm{H}],(\mathbf{3})$, 2,26[s,6H], (4), 2,24[s,6H]; (-N-CH -$),(\mathbf{1}), 4,05[\mathrm{~s}, 2 \mathrm{H}],(\mathbf{2})$, $4,84[\mathrm{~s}, 2 \mathrm{H}],(\mathbf{3}), 3,48[\mathrm{~s}, 2 \mathrm{H}],(\mathbf{4}), 3,87[\mathrm{~s}, 2 \mathrm{H}]$; hidrogênios aromáticos, (1), 7,20-7,80 [m,5H], (2), 7,20-7,60 [m,5H], (3), 7,20-7,46 $[\mathrm{m}, 5 \mathrm{H}],(4), 7,20-7,60[\mathrm{~m}, 5 \mathrm{H}]$; (RMN de $\left.{ }^{13} \mathrm{C}\right), \delta$ em ppm, [-N( $\left.\left(\mathrm{CH}_{3}\right)_{2}\right],(2), 49,74,(3), 47,88,(4), 50,07$; (-N-CH-$),(2), 72,25$, (3), 69,89, (4), 72,05, Pd-C, (2), 148,31, (3), 135,96, (4), 137,37. Não foi medido o espectro de ${ }^{13} \mathrm{C}\left\{{ }^{1} \mathrm{H}\right\}$ do composto $\mathbf{1}$ devido a sua baixa solubilidade em $\mathrm{CDCl}_{3}-d$.

Sugere-se para todos os complexos sintetizados uma estrutura monomérica, com o átomo de paládio apresentando uma geometria de coordenação essencialmente quadrado-planar, ao qual estão coordenados os grupos dmba através de uma ligação sigma Pd-C, os ligante cloro ou pseudohaletos de forma terminal, e a difosfina dppp formando um quelato, conforme mostrado no Esquema 1.

Os compostos 1-4 foram, então, empregados na ativação dos macrófagos in vitro. Conforme já explicitado, foram realizadas medidas de concentração de $\mathrm{H}_{2} \mathrm{O}_{2}$ liberadas pelos macrófagos, empregando-se o método espectrofotométrico e o zimosan como padrão positivo.

Na Figura 2 são mostrados os resultados para os complexos 14; cada barra representa o valor médio obtido em 3 animais e os

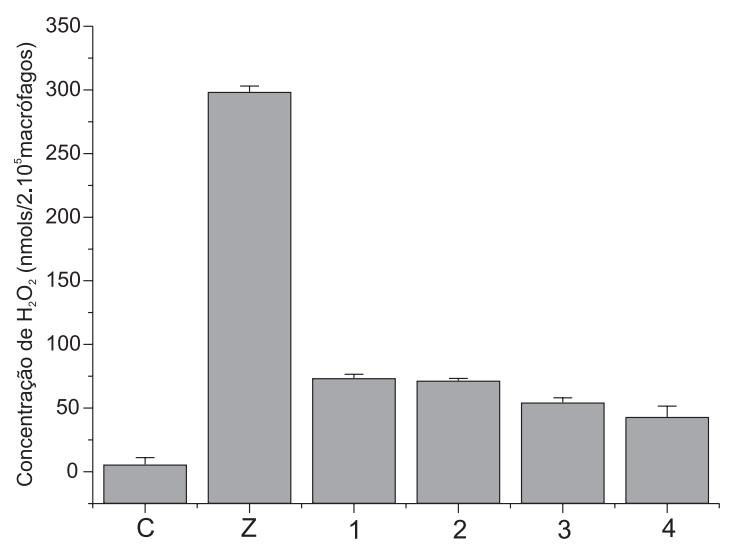

Figura 2 - Concentrações de $\mathrm{H}_{2} \mathrm{O}_{2}$ obtidas em culturas de macrófagos peritoneais de camundongos estimuladas pelos complexos 1-4., $C=$ grupo de controle, macrófagos cultivados em DMSO, $Z=$ zimosan 
testes foram realizados em quadruplicata, sendo que cada pequeno traço acima das barras representa o desvio padrão. O zimosan, parede celular de $S$. cerevisiae, foi utilizado como padrão positivo por provocar uma liberação de $\mathrm{H}_{2} \mathrm{O}_{2}$ da ordem de $300 \mathrm{nmol}$ para cada $2.10^{5}$ macrófagos, em média.

Todos os complexos testados estimularam os macrófagos a liberar $\mathrm{H}_{2} \mathrm{O}_{2}$, sendo a dose resposta inferior àquela provocada pelo zimosan, segundo os dados da Figura 2. Sugere-se que este fato decorra quer pela decomposição de $\mathrm{H}_{2} \mathrm{O}_{2}$ mediada pelo paládio, como por possíveis reações entre $\mathrm{H}_{2} \mathrm{O}_{2}$ e os anéis aromáticos presentes na molécula dos organopaladados empregados ${ }^{20}$.

Considerando que os complexos são estruturalmente similares, ou seja, tratam-se de espécies quadrado-planares mononucleares de $\mathrm{Pd}(\mathrm{II})$, nas quais se variou apenas a presença do pseudo-haleto coordenado ao centro metálico, foi possível verificar a influência destes ligantes na quantidade de $\mathrm{H}_{2} \mathrm{O}_{2}$ liberada e detectada. Assim, a partir dos dados da Figura 2 resulta a seguinte ordem decrescente:

$$
\begin{aligned}
& {[\mathrm{Pd}(\mathrm{dmba})(\mathrm{NCS})(\mathrm{dppp})](4)<[\mathrm{Pd}(\mathrm{dmba})(\mathrm{NCO})(\mathrm{dppp})](3)} \\
& <\left[\mathrm{Pd}(\mathrm{dmba})\left(\mathrm{N}_{3}\right)(\mathrm{dppp})\right](2)<[\operatorname{Pd}(\mathrm{dmba})(\mathrm{Cl})(\mathrm{dppp})](1)
\end{aligned}
$$

Portanto, o composto 4 contendo o pseudo-haleto NCS coordenado de forma monodentada, via átomo de nitrogênio, provocou a menor liberação de peróxido de hidrogênio. As concentrações de peróxido de hidrogênio liberadas por todos os compostos se constituem em resultados excelentes, sendo seus valores de 4 a 7 vezes menores que aquele liberado pelo padrão zimosan. Este fato é de grande importância, pois quaisquer valores de concentração de $\mathrm{H}_{2} \mathrm{O}_{2}$ próximos àqueles produzidos pelo zimosan devem ser tratados como concentrações tóxicas e sua aplicação deve ser avaliada com atenção, em função da aplicação desejada, pois sabe-se que $\mathrm{H}_{2} \mathrm{O}_{2}$ em excesso no organismo pode destruir estruturas biológicas e provocar lesões irreversíveis ${ }^{11}$.

Os resultados mostram que os referidos compostos podem auxiliar o organismo hospedeiro na resposta imunológica, atuando como organo-comunicadores.

Uma consequiência importante advinda deste trabalho reside na possibilidade de se modular a atividade de macrófagos, potentes células imunológicas, mediante variações tanto em $\mathrm{X}$ quanto no fragmento orgânico coordenado ao átomo de paládio.

Finalmente, uma vez determinado que os níveis de concentração de peróxido de hidrogênio liberados no "burst" respiratório não atingem valores considerados tóxicos, há um grande estímulo na continuidade das investigações de outras potencialidades biológicas destes compostos, bem como de outras séries de organopaladados. As investigações que correlacionam a estrutura dos complexos com a ativação dos macrófagos ainda estão em andamento, visando estabelecer os prováveis mecanismos envolvidos.

\section{AGRADECIMENTOS}

Os autores agradecem ao CNPq, CAPES e FAPESP pelo suporte financeiro e à Sra. M. C. P. Placeres pelo auxílio nos ensaios biológicos.

\section{REFERÊNCIAS}

1. Yatsimirsk, A. K.; Kanzakov, G. M.; Ryabov, A. D.; J. Chem. Soc., Perkin Trans. 2 1992, 8, 1295; Camargo, M.; Dani, P.; Dupont, J.; De Souza, R. F.; Pfeffer, M.; Tkatchenko, I.; J. Mol. Catal. A: Chem. 1996, 109, 127; Herrmann, W. A.; Böhm, V. P. W.; Reinsinger, C. P.; J. Organomet. Chem. 1999, 576, 23.

2. Chorodowski-Kimmes, S.; Beley, M.; Collin, J. P.; Sauvage, J. P.; Tetrahedron Lett. 1996, 37, 2963; Carina, R. F.; Williams, A. F.; Bernardinelli, G.; J. Organomet. Chem. 1997, 548, 45.

3. Ghedini, M.; Pucci, D.; Cesarotti, E.; Antonigzza, P.; Francescangeli, O.; Bartolino, R.; Chem. Mater. 1993, 5, 883; Espinet, P.; Esteruelas, M. A.; Oro, L. A.; Serrano, J. L.; Sola, E.; Coord. Chem. Rev. 1995,117, 215; Saccomando, D. J.; Black, C.; Cave, G. W. V.; Lydon, D. P. ; Rourke, J. P.; J. Organomet. Chem. 2000, 601, 305.

4. Higgius, J. D.; J. Inorg. Biochem. 1993, 49, 149; Navarro-Ranninger, C.; López-Solera, I.; González, V. M.; Pérez, J. M.; Alvarez-Valéz, A.; Martin, A.; Raibithby, P. R.; Masaguer, J. R., Alonso, C.; Inorg. Chem. 1996, 35, 5181.

5. Ananias, S. R.; Mauro, A. E.; J. Braz. Chem. Soc. 2003, 14, 764; Ananias, S. R.; Mauro, A. E.; Santana, A. M.; de Lucca Neto, V. A.; de Almeida, E. T.; Quim. Nova 2003, 26, 53; Santana, A. M.; Godoy Netto, A. V.; De Almeida, E. T.; Mauro, A. E.; De Souza Jr., J.; Santos, R. H. A.; Z. Kristallogr. NCS. 2002, 217, 147; de Almeida, E .T.; Santana, A. M.; Mauro, A. E.; Ecl. Quím. 2002, 27, 201.

6. Quiroga, A. G.; Pérez, J. M.; Montero, E. I.; Masaguer, J. R.; Alonso, C.; Navarro-Ranninger, C.; J. Inorg. Biochem. 1998, 70, 117.

7. De Almeida, E. T.; Santana, A. M.; Godoy Netto, A. V.; Mauro, A. E.; Santos, M. P.; Santos, R. H. A.; Z. Kristallogr. NCS. 2002, 217, 366.

8. Caires, A. C. F.; de Almeida, E. T.; Mauro, A. E.; Hemerly, J. P.; Valentini, S. R.; Quim. Nova 1999, 22, 329.

9. Janeway, C. J.; Imunobiologia: o sistema imunológico na saúde e a na doença, $4^{\text {a }}$ ed., Ed. Artes Médicas Sul Ltda: Porto Alegre, 1999.

10. Pick, E.; Keisari, Y.; J. Immunol. Methods 1980, 38, 161.

11. Ramasarma, T.; Indian J. Biochem. Biophys. 1990, 27, 269.

12. Lehninger, A. L.; Nelson, D. L.; Cox, M. M.; Principles of Biochemistry, $2^{\text {nd }}$ ed., Worth Publishers, Inc.: New York, 1993.

13. Ma, J. F.; Yamamoto, Y.; Inorg. Chim. Acta 2000, 299, 164.

14. De Lucca Neto, V. A.; Mauro, A. E.; Caires, A. C. F.; Ananias, S. R.; De Almeida, E. T.; Polyhedron 1998, 18, 413.

15. Moreira, R. R. D.; Carlos, I. Z.; Vilegas, V.; Biol. Pharm. Bull. 2001, 2, 201.

16. Carlos, I. Z.; Zini, M. M. C.; Sgarbi, D. B. G.; Angluster, J.; Alviano, C. S.; Silva, C. L.; Mycopathologia 1994, 127, 189.

17. Pick, E.; Mizel, D.; J. Immunol. Methods 1981, 46, 211.

18. Microcal Software Inc.; Microcal ${ }^{T M}$ Origin $^{T M}$ versão 5,0; Data analysis and Technical Graphics; One Roundhouse Plaza, Northampton, MA, E.U.A., 1997.

19. Nakamoto, K.; Infrared and Raman Spectra of Inorganic and Coordination Compounds - Part B: Applications in Coordination, Organometallic, and Bioinorganic Chemistry, $5^{\text {th }}$ ed., John Wiley \& Sons Inc: New York, 1997.

20. Eberhardt, M. K.; Trends in Organic Chemistry 1995, 5, 115. 